\title{
[1508] Das Verb „brauen“ im Helgoländischen: mit Beibehaltung der starken Flexion und reich entwickelter Semantik
}

Nils Århammar

\begin{abstract}
The verb „brew “ in the North Frisian dialect of the North Sea island of Heligoland is in two respects remarkable: first it has retained its original strong inflection (section 1) and secondly it developed a number of special meanings during the 19/20th century (section 2). I have tried to demonstrate how this great diversity came about: The starting point for the semantic development was probably the analogy 'brew kettle' (for beer brewing) 'steam boiler' (of steam-boats). In a small seafaring nation a shift of meaning from 'brew (beer)' $\rightarrow$ 'drive (a ship)' may seem rather natural; less so the further steps via *'move in general' $\rightarrow$ 'walk briskly, run' and so on ( $\rightarrow$ 'live, keep house, manage to get on well (as a single)' and 'be busy, work' etc.). Sense 8. of the dictionary entry, a figurative meaning, stands apart, namely 'concoct, contrive, prepare, bring about, cause': spec. evil, mischief, trouble, woe (OED). It is noted that this sense was developed in most Germanic languages and it probably represents a much older sprout on the helig. brau-tree than do the senses 2. to 7. In section 3, I deal with the helig. idiom Bin brau 'to bring into disorder' and the helig.-wfris. parallel uun Bin yn 'e/'t bûn 'in disorder'. - In the appendix the revised and enlarged word article brau with its prefix compounds is presented.
\end{abstract}

\section{Morphologie}

Dass es sich bei „brauen“ um ein von Haus aus starkes, ablautendes Verb handelt, dessen dürften sich die wenigsten Germanisten, Anglisten, Frisisten usw. bewusst sein. Denn in den germanischen Nationalsprachen flektiert es in der Neuzeit durchweg schwach, „regelmäßig” (vgl. Grimm 2, 321 und Seebold 1970, 143). Am längsten, d. h. bis in die Gegenwart, konnte sich die starke Flexion m. W. nur im Helgoländischen behaupten. Im ebenfalls isolierten emsfriesischen Dialekt des Saterlandes sind allerdings Reste der starken Flexion überliefert (s. Kramer 1992, 247), während Fort $(2015,102)$ ausschließlich die schwache Flexion verzeichnet. - Saterl. b(r)joue hat übrigens nur die eine Bedeutung 'Bier brauen'. Bereits Siebs $(1893,399)$ berichtet: „Früher baute ein jeder wenigstens soviel davon (an; scil. Gerste), Us Wurk 69 (2020), s. 105-114; https://doi.org/10.21827/5fb7c8c82fc0a 
dass er für seinen Hausbedarf das Bier brauen konnte (bin $\bar{o} r$ bi $\bar{o}^{u}$ ə); das hat aber längst aufgehört."

Ganz parallel zur Flexion von helg. brau - Prät. bruw (auch brou) und Part. Perf. bruwwen - verläuft die von helg. hau 'hauen': Prät. huw (auch hou) und Part. Perf. huwwen. Hier dürften sich die starken Formen der beiden Verba gegenseitig gestützt haben. Im Westerlauw. Friesisch, im Wangeroogischen und Sylterfriesischen ist von „hauen“ nur schwache Flexion überliefert. Siebs $(1901,1322)$ nennt die starke Flexion von saterl. hóô „bemerkenswert“. Der festlandnordfriesische Hauptdialekt, das Mooringer Frasch, hat nach dem FUB $(1988,102)$ schwache und starke Flexion, wobei die in eckigen Klammern stehenden Formen houf ( $f$ analog nach grouf 'grub') und hauen veraltet sein werden. Bendsen $(1860,303)$ gibt noch hau und hauen. ${ }^{1}$ Im Föhring-Amring flektiert hau/haw 'hauen, mähen' nach dem FÖW (2002, 215) schwach, während Wilts (2011, 172) alternativ auch starkes Part. Perf. hauen und (237) für 'gemäht' lediglich diese Form verzeichnet. Es sei daran erinnert, dass bereits in der Altföhrer Ballade das schwache Prät. haud/hawd steht: an haud a saster at hood diar uf 'und schlug („hieb/haute“) der Schwester den Kopf ab'; zitiert nach FÖW (a. a. O.).

\section{Semantik}

Von allen friesischen Dialekten hat nur das Helgoländische eine über die ursprüngliche Bedeutung 'Bier brauen' und den im Anhang unter 8. Behandelten alten figurativen Gebrauch hinausgehende Semantik aufzuweisen. Dabei sind 2. 'fahren (von Dampfschiffen/Motorbooten ${ }^{2}$ usw.)' und die daraus im Laufe des 19./20. Jh.s entwickelten Bedeutungen 3. bis 7. als einmalig zu bezeichnen. Wie unter 2. des Wortartikels angedeutet, dürfte sich die erstgenannte Bedeutung durch Übertragung des Bierbrauens im Braukessel auf den Dampfkessel der Dampfschiffe erklären; diese konnte ja bei

1. Ernst Löfstedt $(1971,105)$ behandelt die Flexion von „hauen“ im Festlandnordfriesischen. Starkes Prät. und Part. Perf. hat er auch für die mittelgoesh. Mda. von Bohmstedt notiert. Bendsens Prät. hau (zunächst < *hô?) bleibt nach Löfstedt dunkel (< mnd. hou, hûw?). - Eine lauthistorische Parallele zu helg. bruw(wen) und huw(wen) bietet helg. buwwe 'bauen'.

2. Vgl. Århammar, Helg. Rudder - vom Ruder- zum Motorboot, im „Helgoländer“ Nr. 450 (Dez. 2001), 47. H. Fr. Heikens (1840): En Sieler off en Rudder? „Ein Segler oder ein Ruderer?"،, kurz für Sieler- bzw. Rudderbooat zu siile 'segeln ' und $r u(d)$ 'rudern'. Bei der Motorisierung der Börteboote ab 1910 wurde die Bezeichnung Rudder beibehalten. 
einem seefahrenden Völkchen wie dem helgoländischen leicht erfolgen. Der Übergang des transitiven ,(Bier) brauen“ zum intransitiven brau 'fahren' ist bei der semantischen Nähe zwischen „ein Dampfschiff fahren“ und „mit demselben fahren" begreiflich. In einem nächsten Schritt fand dann die Ausdehnung auf das Fahren mit Motorbooten und anschließend auf andere Fahrzeuge mit Motorantrieb statt, und in einem darauffolgenden Schritt trat via 'sich fortbewegen allg.' Bedeutungserweiterung zu 'zügig gehen, laufen' (3.) ein. In weiteren Schritten entwickelten sich die unter 4. bis 6 . angeführten Bedeutungen. Die transitive Bedeutung von 7. könnte sich aus der Beförderung mit einem E-Karren, alternativ schon durch den zügig erfolgten Transport mit einer Schubkarre entwickelt haben. ${ }^{3}$

Die Bedeutung 'verursachen, stiften' (8.) schließlich hat vermutlich alte, weiter verbreitete Wurzeln. Die beste Umschreibung dieses Sachverhalts findet sich neuerdings in dem Artikel brouwen I im WNT (Internet), wo es unter 3. heißt:

„Vanouds werden bij de Germanen woorden als brouwen, blanden en schenken veelal in figuurlijken zin gebezigd voor: Toebereiden, klaarmaken, en zoo teweegbrengen, veroorzaken, uitrichten, bewerken, een plan smeden; meestal ten nadeele van een ander.“

Dieselbe Quelle gibt für mnl. brouwen unter 2. die folgenden Bedeutungen: „Beramen, uitdenken, op het touw zetten, overleggen; ook veroorzaken, in de hand werken. Vgl. onze uitdr. kwaad, onheil brouwen, enz.“ ${ }^{4}$ Das WFT

3. Mungard $(1909,45)$ gibt zu sylterfries. bruu neben 1. 'Bier brauen' auch 2. 'etwas sich entwickeln lassen'. Ob dieses zu helg. 6. brau lat 'gewähren lassen' gehört oder eine andere Bedeutung hat, lässt sich bei Ermangelung von Satzbeispielen nicht entscheiden. - Im „Thesaurus des Nordfriesischen: Sylter Friesisch“ (Internet) der Nordfriesischen Wörterbuchstelle an der Universität Kiel (Leitung Prof. Dr. Jarich Hoekstra) ist eine große Anzahl sylterfries. Texte (mit Passwort) abrufbar, darunter die beiden Hauptwerke von Jap Peter Hansen (1767-1855) und die zahlreichen Theaterstücke von Erich Johannsen (1862-1938). In den letzteren finden sich (mittels Browsersuche) die folgenden Beispiele mit übertragener Bedeutung von bruu und Präfixkomposita, die übrigens allesamt in den sylterfries. Wörterbüchern fehlen. Jü bruut nïn Löögens muar! 'Sie „braut“ keine Lügen mehr!' Dreimal Formen von törochtbruu „,zurechtbrauen“, das einmal mit ütbrörre ,ausbrüten“ variiert wird. Ferner Höm grögelk fuul Ünsaachthair töhopbruu let. 'Sich gräulich viel Zwietracht säen (,zusamenbrauen“) lässt.' Und schließlich ja haa wat ön Bruu 'sie haben etwas (Böses) vor' (,im Brau“), hier von Strandräubern. - Die genannten Verben sind alle transitiv und gehören somit zu dem älteren Bedeutungszweig 8 .

4. Ferner s. v. backen 3) evenals ,brouwen“: beramen, berokkenen, (iemand iets) bakken. Vgl. ndl. iem. een poets bakken 'jmd. einen Streich spielen' = westerlauw. fries. immen 
3, 242f. verzeichnet s. v. brouwe I unter 4. die Bedeutung in het geheim beramen, uitdenken' mit der festen Verbindung kwea brouwe und unter 5. 'veroorzaken, berokkenen'.

$\mathrm{Zu}$ 8. gehören bedeutungsmäßig vermutlich auch die präfigierten Verben apbrau und toopbrau (vgl. Anhang).

\section{Helg. Bin brau 'Unordnung schaffen'}

Das helg. Verb brau hat eine feste Verbindung Bin brau 'Unordnung schaffen', neben der das Idiom alles uun Bin 'alles durcheinander' steht (vgl. brau 8.). ${ }^{5} \mathrm{Zu}$ diesem Bin (< *bün < althelg. *bünd) „Bund“ bietet das Westerlauw. Friesisch eine genaue Parallele: WFT 3, 274 bûn II 'Verwarring, verwarde toestand' in der festen Verbindung yn 'e/'t bûn. Zur Etymologie von bûn II verweist der zuständige WFT-Redakteur auf das Zeitwort bauje 'bijzen, wild heen en weer bewegen van koeien door de wei' ${ }^{6}$ unter

in pots / in (raar) koekje bakke (,ein [übles] Küchlein backen“). Grimm 1, 1066 s. v. backen 8) redensarten: ...; was einer nicht backt, das braut der andre. - Vgl. auch die einschlägigen Bedeutungsumschreibungen zu engl. brew im Abstract.

5. Im helg.-dt. Missingsch, genannt „Gätkes Friim“ ( $<$ Gätkes Frem = Dt.), sagt man alles im Bund. Vgl. Århammar N. u. R., Dt.- Helg. Wb. (Internet) s.v. Missingsch.

6. Dies ist eine Ableitung von bau 'Viehbremse/horzel, paardevlieg', welches Etymon ich in dem in Arbeit befindlichen Aufsatz „Ostfries.-nd. Bau, Schuurschott und Tiek(e) drei Insektennamen aus dem friesischen Substrat" behandle. - Zur Entstehung der Bedeutung 'Durcheinander, Unordnung' ist wohl am ehesten an „Bund“ im Sinne von 'Bündel, facies' zu denken. Dieses liegt vor in helg. en Bin Stri 'ein Bund Stroh', en Bin Sleeteler 'ein B. Schlüssel/Schlüsselbund', en Bin Spriin'n 'ein Schwarm Stare' (wurden mit einer Schrotflinte geschossen und gebündelt; beliebt war de Spriinsup 'die Starensuppe'). Ferner in Do hat ii Mem is toopriiwet uun en Bin, ... 'Da hat meine Mutter uns in einen B. zusammengerafft, ...'. Feste Verbverbindungen sind: uun Bin wees (,sein“) / schtjit (,schießen“) / bring (,bringen“) sowie uun Bin (auch Tek ,Zück“) keem 'sich verheddern'. Diese treten bzw. traten besonders auf in Verbindung mit Garn(knäueln), Vögeln im Vogelfangnetz und nicht zuletzt den Angelhaken (Hukker) der Langleine (um Letzteres zu verhindern legte man diese in eine mit feuchtem Sand gefüllten Mulde (Oolsk) bzw. setzte sie auf ein Holzstück mit Scharten (Splets)). In Jo Daan, Wieringer Land en Leven in de Taal (Alphen aan den Rijn 1950, S. 141) finde ich den folgenden Satz im Zusammenhang mit dem Entfernen der Fische aus dem Netz: „Zit er ' $n$ bòs of 'n bongd vis in, dan kan het gebeuren, dat ze door het rondvliegen van de vissen onklaar sitte, er zit dan een ties in [= 'ze zitten in de war']." Wier. bongd zeigt Velarisierung -nd > -ngd, vgl. engd 'Ende'. - Helg. Bin (deät) 'Durcheinander, Unordnung' tritt auch als Subjekt und Objekt auf: Sek en Bin es hiir do ni alle Dai. 'Solch ein D. ist hier ja nicht jeden Tag.' En Stoad, wi hoa deät Bin en ii Fraihait geneeten. 'Eine Pracht, wir haben das D. und unsere Freiheit genossen (bei der 
Berufung auf Epkemas Gysbert-Japicx-Wörterbuch (1824), 63 s. v. Boun, wo das Idiom roun ijn 't boun 'rond in 't wild, volkomen yn de war, als een dolleman' zur Sprache kommt. Diese Zusammenstellung scheitert jedoch an lautlichen Schwierigkeiten. Wie die im WFT angeführten Lautformen bon (Ameland) und bónt (Texel) und erst recht helg. Bin zeigen, kann es sich bei bûn II somit nur um eine Sonderbedeutung von bûn I handeln. Dabei erscheint es unwahrscheinlich, ist aber natürlich nicht ausgeschlossen, dass diese sich in zwei doch in einigem Abstand situierten Sprachen unabhängig voneinander entwickelt haben sollte. Dann wird der Ausgangsherd im Westerlauw. Friesisch mit den friesischen Substratdialekten von Texel und Ameland liegen. Ich habe aber diesen Wortgebrauch im Groninger und dem ostfriesischen Mittelstück nicht belegen können. Wenn er hier nicht im Laufe der Zeit verloren gegangen ist, müssten die Helgoländer ihn im Zuge der Seefahrtverbindungen übernommen haben.

Anhang: Über die Materialgewinnung für das „,Helgoländische Wörterbuch" - Der Wörterbuchartikel helg. brau mit Präfixkomposita

Die dem Wörterbuchartikel zugrundliegende reichhaltige Materialsammlung geht zum allergrößten Teil auf die mit meiner Hauptinformantin Maria Leitgeber-Dähn in den 1970er Jahren gemachten Tonbandaufnahmen und auf ihre eigene Textproduktion zurück (vgl. Århammar 1977, 98f.). Die Tonbandaufnahmen wurden im Auftrag der Mainzer Akademie und später mit Unterstützung des Kieler Kultusministeriums in den 1980er Jahren von meiner Frau Ritva Århammar-Mikkola ausgeschrieben und anschließend größtenteils in eine Datenbank überführt. Daraus kann jedes beliebige Wort mit Kontext für den betreffenden Wörterbuchartikel abgerufen werden.

Als Projektmitarbeiterin im Nordfriisk Instituut in Bräist/Bredstedt schuf meine Frau in den 1990er Jahren ein vorläufiges „Helgoländisch-Deutsches Gebrauchswörterbuch", das noch der Korrektur und Ergänzung harrt. Es wurde in der vorliegenden Form einigen friesischen Institutionen und Interessenten in ausgedruckter Form zur Verfügung gestellt. Der untenstehende Wortartikel ist das Ergebnis einer erneuten, mittels der obengenannten Datenbank vom Verfasser vorgenommenen ergänzenden Bearbeitung.

Auf Bitte der damaligen beiden Helgoländischlehrerinnen hat meine Frau um 1995 mittels automatischer Umkehrung ein „Deutsch-Helgoländisches

Rückkehr auf die verwahrloste Insel nach dem 1. Weltkrieg).' Vgl. auch Bin brau / moake 'U. verursachen'. 
Gebrauchswörterbuch" hergestellt. An dessen Ergänzung vor allem durch die Aufnahme von Redensarten und stets mehr Satzbeispielen habe ich dann seit meiner Pensionierung i. J. 1996 mit der Computerunterstützung meiner Frau in dem Bemühen weitergearbeitet, ein möglichst vollständiges wissenschaftliches Wörterbuch zu schaffen. Dieses noch in Arbeit befindliche „Deutsch-Helgoländische Wörterbuch“ wurde schon vor Jahren zur freien Benutzung ins Internet gestellt (vgl. Århammar 2012 und Århammar, Nils \& Ritva. 1995-). ${ }^{7}$

brau (braut; bruw [auch brou]; bruwwen) 1. Biir brau Bier brauen (Siebs 1909, 204/Krogmann 125 wohl falsch braue; Kgm. sw. V.) 2. fahren (von Dampfschiffen, Motorbooten, Autos u. a. Motorfahrzeugen; urspr. wohl vom Brau- $\rightarrow$ Dampfkessel): de Damper braut, hi komt al iinbrauen der Dampfer kommt angefahren > hinein- (Siebs a. a. O.; richtiger tubrauen „angebraut"); Klok seeben skul de Damper brau um sieben Uhr sollte der Dampfer f.; wi brau noa de Ellow tu wir f. Richtung Elbe; dja mut dan do wel noa de Fastewal tu brau sie müssen dann ja wohl zum Festland f.; dan bruw 'e Rudder oawerlang fiider dann fuhr das Motorboot langsam weiter; en dan bruw man hen oder rut man hen noa de uur Partii Tiiners und dann fuhr man hin oder ruderte man hin zu der anderen/zweiten Partie Hummerkörbe; Seegat it brau in See stechen (,Seeloch hinaus f.“); oostert brau zur Düne(ninsel) f.; rin-om Klef brau um den Felsen herum f.; om Prüs brau um die Wette (,um Preis“) f.; djin 'e Strooam brau gegen den Strom schwimmen. 3. zügig gehen, laufen: hentüs brau nach Hause gehen; boppenap/önner-uf brau nach oben/unten g. (im Hause); weer apperdans brau wieder nach oben g.; hendeel/henboppen brau ins Unter-/Oberland gehen; it iip 'e Bräi brau auf die Landungsbrücke hinausg.; deel bi Strun brau zum Strand hinunterg.; De Muskanten bruw óm om Was. Die Musiker liefen/ marschierten nach dem Westen (des Unterlandes). Kan wi nä dan brau? Können wir jetzt denn g./los? Ik leow, ik mut brau. Ich glaube, ich muss los. Dear kans 'e uuderweegen ni med brau, med deät dear Tschich ni. Damit kannst du auf dem Festland nicht (herum)laufen, mit dieser Kleidung nicht. Vgl. apbrau, dearfandanbrau, feerwarsbrau, fiiderbrau, henbrau, hen- en heerbrau, iinbrau, itbrau, ombibrau, uundjinbrau, wechbrau 4. leben, zurechtkommen, wirtschaften, wohnen: Tu'n Glik kiid dji do no soo alleen

7. Ohne die technische Hilfe meiner Frau auch bei der Herstellung des Druckmanuskripts hätte dieser Aufsatz nicht erscheinen können. - Patrick Stiles, London, danke ich für seine Anregungen, speziell zur Formulierung des Abstracts. - Last but not least gilt mein Dank den redaktionellen Reviewern für konstruktive Kritik und Anregungen. 
brau. Zum Glück konnte sie ja noch alleine zurechtkommen. En djerrem Oot hat do nä al föfti Djooar alleen dear boppen bruwwen. Und deine (,eure“) Großmutter hat jetzt ja schon fünfzig Jahre allein dort oben gelebt/ gewirtschaftet. ..., dan wart dear uk do wel no en betjen spoorsoamer bruwwen. ..., dann wird dort ja wohl noch ein wenig sparsamer gelebt/ gewirtschaftet. Luuma, dja brau nä do man aal soo it Folns. Schaut, sie wirtschaften jetzt ja nur so aus dem Vollen. Dear bruw dja soo noa herrem Glooben fiider. Da lebten sie so nach ihrem (jüdischen) Glauben weiter. Brau man soo fiider! Mach' nur so weiter! Soo hoa wi is deät dan uunwuunt tu brauen mus-meen Mem en Kin. So haben wir es uns dann angewöhnt wie Mutter und Kind zu leben. 5. beschäftigt sein, arbeiten, sich aufhalten/ befinden, spielen, tun und lassen, zusammensein: Herrem Mem bruw önner uun Keeken. Ihre Mutter arbeitete/befand sich unten in der Küche. Med hem kiid ik do soo roor brau. Mit ihm (zusammen) konnte ich ja so gut arbeiten. Wi kiid rech nons soo brau es wi wul. Wir konnten mal so richtig tun und lassen was („wie“) wir wollten. 6. gewähren lassen: Lat djam (man) brau! Lass(t) sie g.!/sie (nur) machen! Dan lat djam man alleen/sallow brau! Dann lass(t) sie man allein/selbst machen! 7. (trans.) schaffen, befördern: $I k$ brau 'et henboppen, deät Gefras. Ich schaffe es ins Oberland, das Zeug/den Krempel. 8. (trans.) verursachen: Bin brau Unordnung v./stiften („Bund brauen"). En nä brau dja hiir alles uun Bin. Und jetzt bringen sie hier alles durcheinander. Skit brau Mist bauen.

apbrau $u$ (trans.) erdichten, erfinden und in Schwang/Umlauf bringen; Gerüchte in U. bringen Stekken (,Stücke“) apbrau. Vgl. apteenk.

beäfuunbrau $u$ hinterherfahren; hinterherlaufen

dearfandanbrau $u$ sich aufmachen, sich auf den Weg machen, sich entfernen, sich fortbegeben, abdampfen: Hi bruw dear do weer fandan. Er machte sich dann wieder auf den Weg.

dearmankbrau $u$ dazwischenfunken, drein-/hineinreden, intervenieren, sich einmischen: Brau is dear do man ni uk no mank! Red' uns ja nur nicht auch noch drein!

feerwarsbrau $u$ vorwärtsfahren: En dan mut djüm med Folstiim f. Und jetzt müsst ihr mit Volldampf $v$.

fiiderbrau $u$ 1. weiterfahren: Dan bruw 'e Rudder oawerlang fiider. Dann fuhr das Motorboot (vgl. dies) langsam weiter. 2. weitermachen: Hi bruw dear aal soo fiider. Er machte da die ganze Zeit so weiter. Vgl. brau 4.

henbrau $u$ (schnell) hingehen, hinlaufen: De Groaw braut hen noa herrem Groofoor. Der Graf geht hin zu ihrem Großvater. De uur Moorn brau ik hen om Rinstekken. Am nächsten Morgen laufe ich hin um Brötchen (zu holen). 
hen- en weerbrau $u$ hin- und herlaufen

iinbrau $u$ 1. hineinfahren: De Damper küm uun 'e Hoawen iinbrauen. Der Dampfer kam in den Hafen hineingefahren. Vgl. brau 2. 2. (schnell) hinein-/ reingehen: Deät Oolsk bruw weer iin. Die Alte ging/lief wieder (ins Haus) rein. Dja bruw en Mooremen medarker iin uun 'e Wald. Sie liefen am Morgen miteinander in den Wald (hinein).

itbrau $u$ 1. hinausfahren: En do sen wi Nathoawen/Seegat itbruwwen. Und dann sind wir den Nordhafen/auf die See hinausgefahren. Vgl. brau 2. 2. (aus dem Haus) hinausgehen: Hi bruw it (feer Deer)/bitten it. Er ging hinaus (vor die Tür).

oawerbrau $u$ (hin)über-/rüberfahren: Dja brau hiir oawer. Sie (die Motorboote) fahren hier (nach Helgoland) rüber.

ombibrau $u$ herumlaufen, herumschwirren, herumtoben/-tollen, herumwuseln: Wi bruw dear no aal mankarker ombi. Wir liefen da noch die ganze Zeit durcheinander herum. Ii Djongen bruw dear do ombi es Skiirskotten uun en Sigaarnkes, dja wul it. Unsere Jungs liefen/schwirrten da ja herum wie Libellen in einer Zigarrenkiste, sie wollten hinaus (zum Spielen). En dan bruw dja dear aal önner Klef ombi. Und dann liefen sie da die ganze Zeit unter dem Felsen herum. Dja brau dear uun alle Dörnsken ombi. Sie laufen dort in allen Zimmern herum.

toopbrau $u$ (trans.) (schnell) zusammenkochen (engl. concoct): Ii Mem bruw ii betjen Iiten soo gau toop en futtert is uun 't Baad. Meine (,unsere“) Mutter kochte unser bisschen Essen so schnell zusammen und fütterte uns im Bett. Vgl. toopwiite (,zusammenwüten“).

uundjinbrau $u$ entgegenfahren; entgegenlaufen

wechbrau $u$ wegfahren

Gebrau (deät) 1. Gehaben, Verhalten: Miin Moats en din'n herrem Memmen din'n kiid deät likkers ni kloore, wat deät Gebrau skul. Meine Freundinnen und deren Mütter konnten es so wie so nicht verstehen, was das V. (meiner Eltern) sollte. 2. Gebahren, Tun und Treiben: Soo seät ik dan iip ii Tante Nan her Drumpel en skiilt fan Fiirn noa herrem Gebrau. So saß ich dann auf der Schwelle von unserer Tante Anna und schielte aus der Ferne nach ihrem G. (der Badegäste, die vor dem Nachbarshaus am Frühstücken waren). 3. Wirtschaften, Lebensweise: Deät wear no rech oolung Halunder Gebrau. Es war (herrschte im Hause der Fam. M.) noch so richtig altmodisches Helgoländer W.

Gerichtstraße 60

D-25821 Bredstedt

Arhammar@t-online.de 


\section{LITERATURVERZEICHNIS}

Århammar, Nils. 1977. Die Sprache Helgolands und ihre Erforschung. In: Philologia Frisica anno 1975. Ljouwert: Fryske Akademy, 92-100.

Århammar, Nils. 2012. Das Helgoländische (Deät Halunder): Stand und Perspektiven seiner Erfassung, Erschließung und Erforschung. In: Philologia Frisica anno 2008. Ljouwert: Fryske Akademy, 133-150.

Århammar, Nils \& Ritva. 1995-. Deutsch-Helgoländisches Wörterbuch. Bredstedt: Im Selbstverlag. www.helgolaendisch-halunder.de

Bendsen, Bende. 1860. Die nordfriesische Sprache nach der Moringer Mundart. Hrsg. von M. de Vries. Leiden: E. J. Brill.

Fort, Marron Curtis. 2015. Saterfriesisches Wörterbuch. Hamburg: Helmut Buske.

FÖW = Fering-Ö̈̈mrang Wurdenbuk. 2002. Wörterbuch der friesischen Mundart von Föhr und Amrum. Hrsg. von der Nordfriesischen Wörterbuchstelle der Christian-Albrechts-Universität Kiel. Neumünster: Wachholtz.

FUB $=$ Frasch Uurdebök. 1988. Wörterbuch der Mooringer Mundart. Zusammengestellt von Bo Sjölin, Alastair G. H. Walker, Ommo Wilts. Neumünster: Wachholtz.

Grimm, Jacob und Wilhelm. 1860. Deutsches Wörterbuch. Zweiter Band. Leipzig: S. Hirzel.

Kramer, P. 1992. Näi Seelter Woudebouk. Neues Saterfriesisches Wörterbuch. Nij Sealter Wurdboek. New Saterfrisian Dictionary. I. (A-E). Elst: Im Selbstverlag.

Krogmann, Willy. Helgoländer Wörterbuch. A-L (1957-1967). Mainzer Akademie der Wissenschaften und der Literatur, Band I. Wiesbaden: Franz Steiner.

Löfstedt, Ernst. 1971. Beiträge zu einer nordfriesischen Grammatik II. Das Pronomen, das Adverb, Präpositionen, Konjunktionen und das Verbum. Uppsala: Almqvist \& Wiksell.

Mungard, Nann. 1909. For Sölring Spraak en Wiis. Eine Sammlung von Sylter Wörtern, ... Keitum a. Sylt: Sölring Foriining.

OED = The Compact Edition of the Oxford English Dictionary. Vol. I/II (1971). Oxford University Press.

Seebold, Elmar. 1970. Vergleichendes und etymologisches Wörterbuch der germanischen starken Verben. The Hague / Paris: Mouton. 
Siebs, Theodor. 1893. Das Saterland. Ein Beitrag zur Deutschen Volkskunde. Sonderdruck aus der Zeitschrift des Vereins für Volkskunde. Heft 3, 239-410.

Siebs, Theodor. 1901. Geschichte der friesischen Sprache. In: Paul, Hermann (Hrsg.), Grundriss der germanischen Philologie. Bd. I². Strassburg: Trübner, 1152-1464.

Siebs, Theodor. 1909. Helgoland und seine Sprache. Beiträge zur Volksund Sprachkunde. Cuxhaven / Helgoland: Aug. Rauschenplat.

WFT $=$ Wurdboek fan de Fryske taal. Woordenboek der Friese taal 1-25 (1984-2011). Fryske Akademy. Ljouwert / Leeuwarden: De Tille.

Wilts, Ommo. 2011. Wörterbuch Deutsch-Föhrer Friesisch. Westerlandföhr. Insel Amrum: Jens Quedens.

WNT $=$ Woordenboek der Nederlandsche Taal. (Internet) Historische Woordenboeken, Instituut voor Nederlandse taal. Geïntegreerde Taalbank: www.gtb.inl.nl 